\title{
The Design of SOA-based Multiwavelength Fiber Ring Laser for Fiber Sensing Network
}

\author{
Zhifeng Sun, Deming Liu, Hairong Liu \& Qizhen Sun \\ College of Optoelectronic Science and Engineering \\ Huazhong University of Science and Technology, Wuhan 430074, China
}

$\&$

National Engineering Laboratory for Next Generation Internet Acess System, Wuhan 430074, China

$\&$

Wuhan National Laboratory for Optoelectronics, Wuhan 430074, China

E-mail: dmliu@mail.hust.edu.cn

\begin{abstract}
A SOA-based multiwavelength fiber ring laser for fiber sensing network is introduced in the paper. According to the demand of our sensing network which is a six-channel WDM system, the output wavelength of our MFRL is in the band of $1537 \mathrm{~nm}$ to $1547 \mathrm{~nm}$ with a frequency space of $200 \mathrm{GHz}$. In the experiments, stable output and good flatness are acquired. Factors that can influence the wavelength shift of the laser output are also discussed and demonstrated.
\end{abstract}

Keywords: Sensing network, SOA-based, Multiwavelength, Fiber ring laser

\section{Introduction}

In recent years, there is a considerable interest in sensing network because traditional single-point sensors are not able to supply the demands of gradually expanding sensing fields. Especially combined with WDM (Wavelength Division Multiplexing) and TDM (Time Division Multiplexing) technology, the capacity of sensing network can be improved significantly. With the introduction of WDM technology in the sensing network, multiwavelength fiber lasers (MFLs), which can provide an efficient and economical solution to increase network's flexibility and reduce their cost, have been attracting more and more attention.(Junqiang Sun, Ying Zhang, Xinliang Zhang, 2002)

MFLs have been widely investigated using various gain media, such as erbium-doped fiberamplifiers (EDFAs)(Sun Guoyong, Qu Ronghui, Yang Jing et.al., 2005), Raman fiber amplifiers (RFAs)(Nam Seong Kim, Xingyu Zou, Kirk Lewis. 2002) and semiconductor optical amplifiers (SOAs). Comparing with other solutions, SOA-based MFLs have several advantages. Firstly, SOAs' dominant property of inhomogeneous broadening can support more wavelength lasing oscillations simultaneously in the laser cavity. On the contrary, because of the homogeneous gain broadening of an erbium ion, it is difficult to realize large numbers of stable multiwavelength oscillations in EDF. Secondly, due to the short carrier transmit time of SOAs, SOA-based MFLs have small relaxation oscillation and excellent super-mode rejection effect, which promise the stable performance of lasers.

A SOA-based multiwavelength fiber ring laser(MFRL) for fiber sensing network is introduced in this paper. According to the demand of our sensing network which is a six-channel WDM system, the output wavelength of our MFRL is in the band of $1537 \mathrm{~nm}$ to $1547 \mathrm{~nm}$ with a frequency space of $200 \mathrm{GHz}$. The linewidth of each wavelength is about $0.5 \mathrm{~nm}$, the extinction ratio is above $30 \mathrm{~dB}$. The power non-uniformity among six wavelength is less than $2 \mathrm{~dB}$.

\section{Experiment Device}

The experimental arrangement for our SOA-based MFRL is schematically shown in Fig1. A SOA with 14dB maximal gain is employed as a gain medium. An optical isolator ensures the unidirectional lasing oscillation. A polarization controller is employed to control the states of polarization and optimize the lasing operation. A $10 / 90$ coupler is introduced to form the ring cavity and used for the laser output. A Fabry-Perot filter (FPF) is employed to acquire a frequency space of $200 \mathrm{GHz}$.

As the gain media of MFRL, the characteristic of the SOA adopted in the design is crucial to the performance of MFRL. Fig 2 shows the spectrum of the amplified spontaneous emission (ASE) light of the SOA at different bias current. The center wavelength of ASE is about $1550 \mathrm{~nm}$ with a $3 \mathrm{~dB}$ bandwidth above $60 \mathrm{~nm}$. Obviously, with the increasing of the bias current, the power of the ASE light is improved and the center wavelength of ASE 
is shifted to short wavelength (Blue shift). Beyond this, When the bias current is increased, the maximum small-signal gain point is also shifted to short wavelength for an input signal power of $-25 \mathrm{dBm}$. Both the ASE and small-signal gain spectrum of this SOA are very flat, that is important for MFRLs to support many laser line oscillations simultaneously.

In addition, it is found that the SOA ripple, Polarized Dependent Gain(PDG) and polarization mode dispersion from components have the significant influence to the power flatness of output. In the design, the ASE ripple of the SOA is less than $0.2 \mathrm{~dB}$ and the PDG is $1 \mathrm{~dB}$ at $1550 \mathrm{~nm}$ when SOA is operated at $500 \mathrm{~mA}$. So it is important to employ a polarization controller in the ring cavity to control the states of polarization.

\section{Experiment Results and Discussion}

Power stability is one of the important parameters of MFRL. SOA has a broad and flat gain spectrum within $50-60 \mathrm{~nm}$ range, and the gain deviation of several lasing wavelengths is small, which can enlarge the output bandwidth of the laser. Due to the small insertion loss of the FPF used in the experiment, the total loss of the ring cavity is relatively small, so it is easy for SOA to operate at the deep saturated condition because of the strong optical feedback in the laser cavity. At the deep saturated condition, it is possible to suppress longitudinal-mode competition to get stable laser output.(Hongxin Chen, 2005)

Another important parameter of MFRL is power flatness. For SOA-based MFRL, the deep saturated condition enhances the nonlinear effect in the ring cavity. With the increasing of nonlinear effect, the gain saturation effect of SOA makes wavelengths at high power to get lower gain and wavelength at low power to get higher gain. Further more, four-wave-mixing(FWM) effect created by multiwavelength oscillations leads the power at one wavelength to be transferred to its adjacent wavelengths(Govind P. Agrawal, Isam M. I. Habbab, 1990). Especially for those wavelengths at higher power, because higher power brings more significant nonlinear effect, more power will be transferred to other wavelengths. Based on the analysis above, the combined action of these two nonlinear effects makes the power at different wavelength in the cavity to achieve auto equilibrium, which can promise that the output of SOA-based MFRL has excellent power flatness.

Fig 3 shows the spectrum of SOA-based MFRL when the SOA is operated at $450 \mathrm{~mA}$. In this highly saturated condition, the laser output is very stable, as are multiwavelength laser output powers. The extinction ratio is above $30 \mathrm{~dB}$. In the range $1537 \mathrm{~nm}$ to $1550 \mathrm{~nm}$, output has good flatness even there is a small variation in the multiwavelength laser output.

In the experiments, when laser is operated at different bias currents, the center wavelength of the spectrum is shifted. Show in Fig 4, with the increasing of the bias current, a wider spectrum is observed and the center wavelength of the output spectrum is also shifted with a small amount. When the bias current is added, the peak gain wavelength is blue shift because of the increasing of the density of the current carriers, but FWM effect in the ring cavity is promoted to lead the output wavelength red shift. These two opposing effects make the variation of the center wavelength small.

Predictably, if an adjustable attenuator is added in the laser cavity, when the loss of the ring cavity is increased, the optical feed back in the cavity is decreased, that enhanced the density of the current carrier in the SOA to make the peak gain wavelength blue shift. At the same time, the FWM effect in the ring cavity is reduced to lead the output wavelength blue shift. Combined with these two factors, the center wavelength of output spectrum is notably shifted to a short wavelength (blue shift).

In order to make the output wavelength within the range needed by the sensing network, a BPF can be added in the ring cavity. With the BPF, the wavelength of lasing oscillations is restrained in the band from $1337 \mathrm{~nm}$ to $1347 \mathrm{~nm}$. In this range, combined with the frequency choosing effect of FPF, the laser output can comfort with the demand of the sensing network.

\section{Conclusion}

A simple multiwavelength fiber ring laser is demonstrated with a SOA and a FPF. Simultaneous multiwavelength lasing is observed with a frequency separation space of $200 \mathrm{GHz}$ which is dependent on the characteristic of FPF. The broadband and flat gain effect of SOA is a determined factor of the stable and broadband multiwavelength lasing oscillations. With the reasonable adjustment of the cavity loss and the bias current, SOA-based MFRL can be designed to tune a wide wavelength range. And, with an adjustable BPF, it can also be a multiwavelength-tuned device. These wavelength-tuned designs are no doubt to be widely adopted in the novel sensing network even other professional fields. 


\section{Acknowledgment}

The work is supported by National Natural Science Foundation of China (Grant No. 60807012 and Grant No. 60937002).

\section{References}

Govind P. Agrawal, Isam M. I. Habbab. (1990). "Effect of four-wave mixing on multichannel amplification in semiconductor laser amplifiers. IEEE J. Quantum Electron. 1990, 26(3).

Hongxin Chen. (2005). "Multiwavelength fiber ring lasing by use of a semiconductor optical amplifier", OPTICS LETTERS, Vol. 30, No.6, March 15, 2005.

Junqiang Sun, Ying Zhang, Xinliang Zhang. (2002). "Multiwavelength Lasers Based on Semiconductor Optical Amplifiers”. IEEE PHONTONICS TECHNOLOGY LETTERS, VOL. 14, NO.5, MAY 2002.

Nam Seong Kim, Xingyu Zou, Kirk Lewis. (2002). “CW Depolarized Multiwavelength Raman Fiber Ring Laser with over 58 Channels and $50 \mathrm{GHz}$ Channel Spacing[C]. Optical Fiber Comunication Conference and Exhibit 2002(OFC 2002), 2002.

Sun Guoyong, Qu Ronghui, Yang Jing et.al. (2005). "Study on stable multi-wavelength erbium-doped fiber laser at room temperature. Acta Optica Sinica, 2005, 25(6).

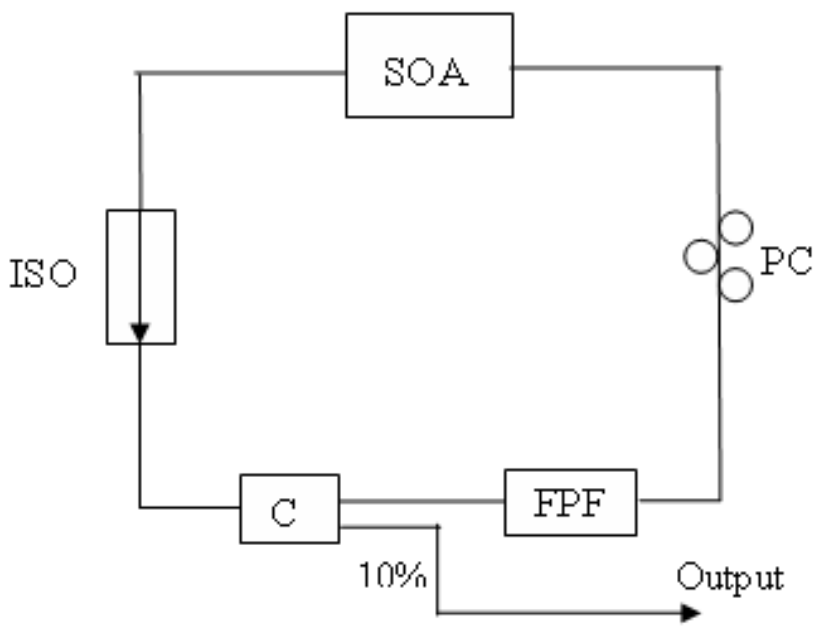

Figure 1. The schematic of SOA-based MFRL

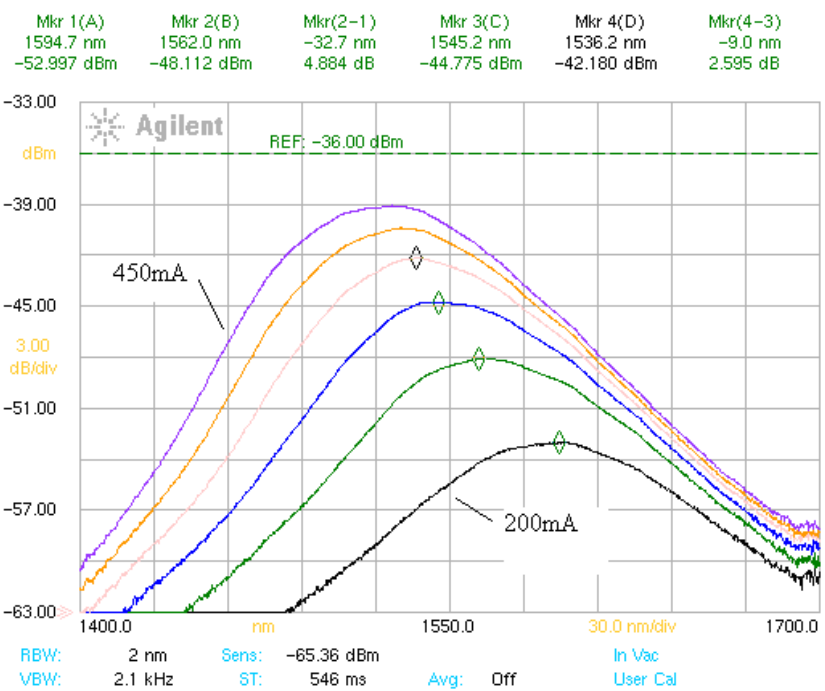

Figure 2. The spectrum of ASE light of the SOA at different bias current 


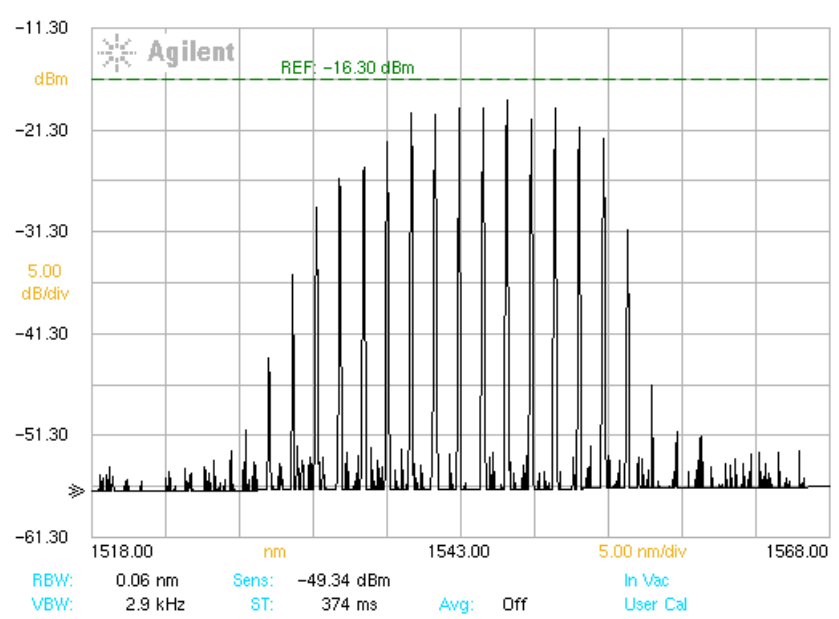

Figure 3. The spectrum of SOA-based MFRL when the SOA is operated at $450 \mathrm{~mA}$

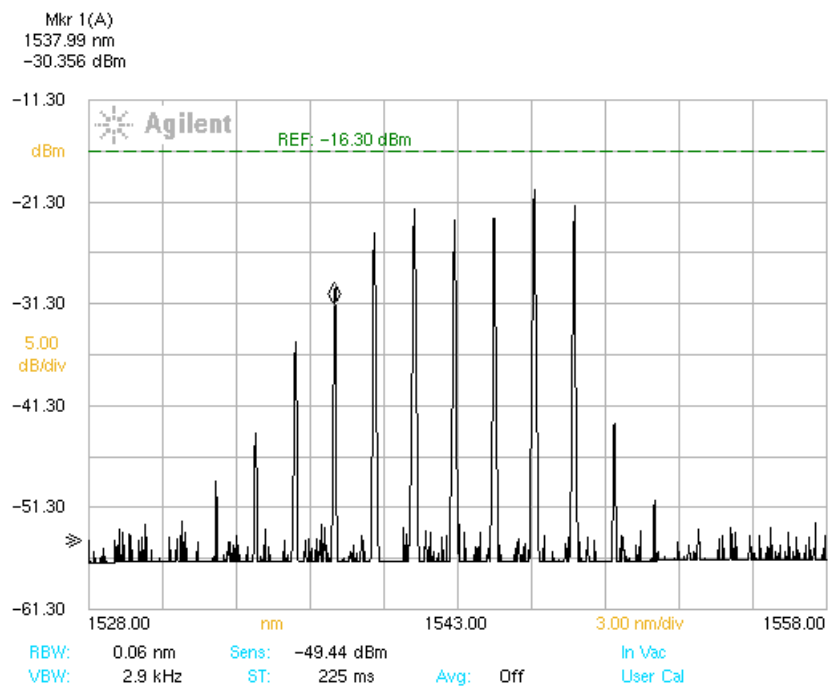

Figure 4 (a). Output of 250mA Bias current.

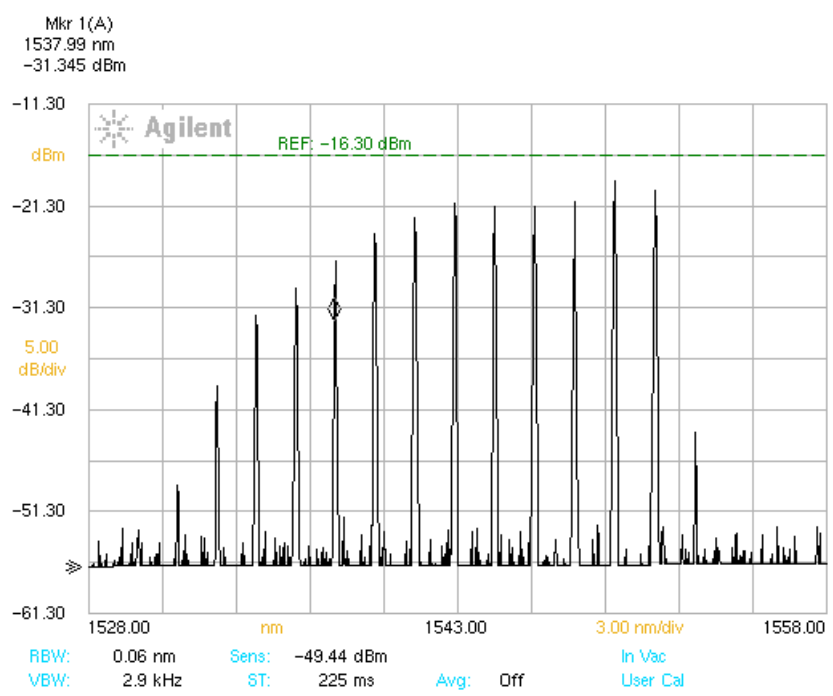

Figure 4 (b). Output of 300mA Bias current. 


\section{Mkr 1(A)}

$1537.99 \mathrm{~nm}$
$-27.120 \mathrm{dBm}$

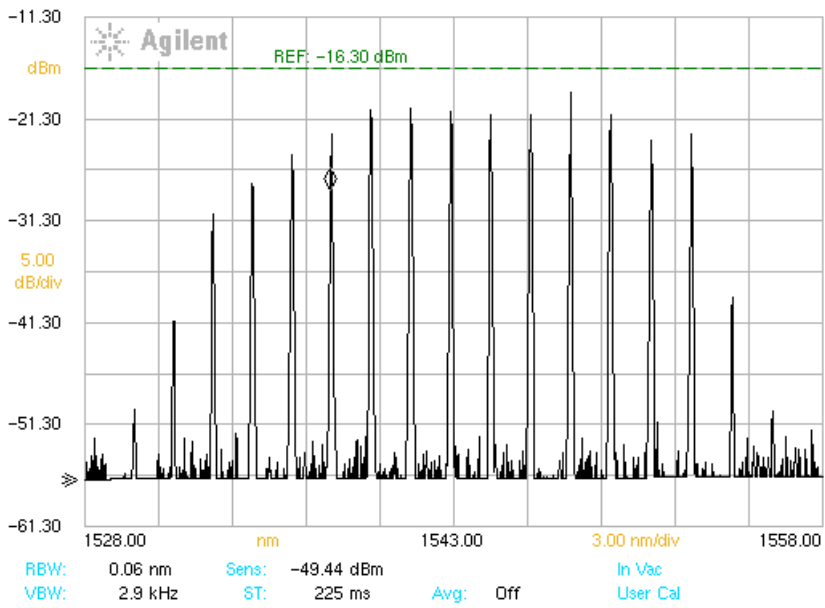

Figure 4 (c). Output of 350mA Bias current. 